\title{
Anisotropic susceptibility of ferromagnetic ultrathin Co films on vicinal $\mathrm{Cu}$
}

\author{
P. J. Jensen* \\ Institut für Theoretische Physik, Freie Universität Berlin, Arnimallee 14, D-14195 Berlin, Germany \\ S. Knappmann ${ }^{\dagger}$ and W. Wulfhekel ${ }^{+}$ \\ Institut für Grenz- und Oberflächenphysik (ISG3), Forschungszentrum Jülich, D-52425 Jülich, Germany \\ H. P. Oepen ${ }^{\S}$ \\ Institut für Angewandte Physik, Universität Hamburg, Jungiusstrasse 11, D-20355 Hamburg, Germany
}

(Received 27 September 2002; revised manuscript received 23 January 2003; published 27 May 2003)

\begin{abstract}
We measure the magnetic susceptibility of ultrathin Co films with an in-plane uniaxial magnetic anisotropy grown on a vicinal $\mathrm{Cu}$ substrate. Above the Curie temperature the influence of the magnetic anisotropy can be investigated by means of the parallel and transverse susceptibilities along the easy and hard axes. By comparison with a theoretical analysis of the susceptibilities we determine the isotropic exchange interaction and the magnetic anisotropy. These calculations are performed in the framework of a Heisenberg model by means of a many-body Green's function method, since collective magnetic excitations are very important in twodimensional magnets.
\end{abstract}

DOI: 10.1103/PhysRevB.67.184417

PACS number(s): 75.30.Gw, 75.40.Cx, 75.70.Ak

\section{INTRODUCTION}

The investigation of the magnetic properties of ferromagnetic ultrathin films is a field of intense current interest. ${ }^{1}$ Among the different experimental methods the measurement of the magnetic susceptibility $\chi(T)$ is a very powerful method for the analysis of such thin film systems. ${ }^{2}$ The singularity of $\chi(T)$ corresponds to the onset of a ferromagnetic state, i.e., to the occurrence of a nonvanishing magnetization $m(T)=|\mathbf{m}(T)|$ for temperatures below the (ferromagnetic) Curie temperature $T_{C}$. For $T \gg T_{C}$ the inverse susceptibility $\chi^{-1}(T)$ exhibits the linear (Curie-Weiss) behavior: $\chi^{-1}(T)$ $\propto T-T_{C}^{\text {para }}$. The paramagnetic Curie temperature $T_{C}^{\text {para }} \geqslant T_{C}$ is obtained from the extrapolation of this linear behavior to $\chi^{-1}(T)=0$, which corresponds to the Curie temperature calculated in the mean field approximation. ${ }^{3}$ For an isotropic ferromagnet the behavior of $\chi(T)$ does not depend on the lattice orientation.

In the collectively ordered magnetic state the direction of the magnetization is determined by magnetic anisotropies, which are the free energy differences between the hard and easy magnetic directions. Due to their relativistic origin resulting from the spin-orbit interaction they are usually much smaller than the isotropic exchange. As obtained in experiments the anisotropies depend on temperature and are expected to vanish above the Curie temperature. ${ }^{4}$ It is known from general considerations ${ }^{5}$ that the mentioned singularity (or maximum) of the susceptibility is only observed if $\chi(T)$ is measured along easy magnetic directions. Corresponding experiments have been performed for bulk systems, ${ }^{6}$ a thin film investigation has been reported for the $\mathrm{Fe} / \mathrm{W}(110)$ system. ${ }^{7}$ Thus, a signature of the anisotropy is also present in the paramagnetic state above $T_{C}$.

At first we comment on the fact that the anisotropy is noticeable also for $T>T_{C}$. We would like to stress the fact that the microscopic anisotropy, e.g., the single-ion uniaxial anisotropy $K_{2}$ as present in a Heisenberg Hamiltonian, is different from the effective, temperature dependent anisotropy $\mathcal{K}_{2}(T)$ as measured for a collectively ordered magnetic state. ${ }^{4}$ The effective anisotropy is equal to the microscopic one for $T=0$, thus $\mathcal{K}_{2}(0)=K_{2}$. When treated as a perturbation to the exchange interaction, $\mathcal{K}_{2}(T)$ indeed vanishes at $T_{C} \cdot{ }^{4}$ However, a vanishing effective anisotropy for $T>T_{C}$ does not indicate that the microscopic anisotropy vanishes either, or that the underlying spin-orbit coupling is strongly varying with temperature. A noticeable drop of the spin-orbit coupling is expected on a larger temperature scale. ${ }^{8}$ Thus, a single magnetic moment in the paramagnetic state is still subject to the anisotropy even if the net magnetization is zero. Here a free energy difference between the easy and hard magnetic directions is also present ("paramagnetic anisotropy"), exhibiting a temperature behavior as $\propto\left(K_{2}\right)^{2} / k_{B} T$ for $K_{2} \ll k_{B} T$, with $k_{B}$ the Boltzmann constant. ${ }^{9}$ Evidently, the paramagnetic anisotropy is rather small as $K_{2}$ is small compared to the exchange interaction $J \propto k_{B} T_{C}$.

In the present study we will show that the anisotropyalthough small—has a sizable effect on the susceptibilities in the paramagnetic state of ultrathin films in particular when approaching the Curie temperature. Whereas a vast amount of susceptibility data are available for various systems, ${ }^{2}$ to our knowledge the different behavior of $\chi(T)$ measured along the easy and hard magnetic directions has not been exploited to gain information about thin films. In this paper we report measurements of $\chi(T)$ for ferromagnetic ultrathin Co film grown on a vicinal $\mathrm{Cu}$ substrate. This system exhibits an in-plane twofold (uniaxial) magnetic anisotropy due to the presence of regularly distributed steps in the $\mathrm{Cu}$ surface, with the easy axis directed along the steps. ${ }^{10}$ We find strong differences for the magnetic susceptibilities along the easy $(z)$ and hard $(x)$ in-plane magnetic directions. With the help of an anisotropic Heisenberg model solved within a manybody Green's function method we are able to perform a quantitative comparison with experiments. Furthermore, we demonstrate how the exchange interaction and magnetic an- 
isotropy can be extracted from these susceptibilities. In the previous study on $\mathrm{Fe} / \mathrm{W}(100)$ films only a qualitative comparison in the framework of a renormalization treatment was possible. ${ }^{7}$ Our present approach represents a new method to study quantitatively magnetic properties of ultrathin ferromagnetic films with the help of high-accuracy susceptibility measurements above $T_{C}$.

The paper is organized as follows. In Secs. II and III we describe the experimental methods and the theoretical model. Results from measurements and calculations are presented in Sec. IV. A discussion and conclusion is given in Sec. V.

\section{EXPERIMENTAL ASPECTS}

The experiments, including film preparation and investigation of the magnetic properties, were performed under ultrahigh vacuum (UHV) conditions (base pressure $\sim 10^{-10}$ Torr) in the same chamber. The film characterization and surface preparation were made via Auger electron spectroscopy (AES), low energy electron diffraction (LEED), and medium energy electron diffraction (MEED).

Vicinal $\mathrm{Cu}(11 \mathrm{n})$ surfaces with $n=17$ are used as templates for the ultrathin Co films. These surfaces have been well studied by means of helium scattering ${ }^{11}$ and scanning tunneling microscopy (STM). ${ }^{12}$ Microscopically, the surfaces consist of terraces with the normal oriented along the (001) direction, and an average terrace width of $n / 2$ atomic distances. The terraces are separated by monoatomic steps which are aligned along the $[1 \overline{1} 0]$ in-plane direction. Step bunching has not been observed. The substrate was cleaned and prepared by cycles of Ar sputtering $(600 \mathrm{eV})$ and subsequent annealing $\left(T>670{ }^{\circ} \mathrm{C}\right)$. The quality of the surface structure was confirmed via LEED and MEED. Pronounced splitting of regular lattice spots were found, indicating the periodic step arrangement on the surface.

The Co films were grown at $T=45^{\circ} \mathrm{C}$ with a rate of one monolayer (ML) per minute. During electron beam evaporation the pressure did not exceed $5 \times 10^{-10}$ Torr. The growth process was monitored by measuring MEED intensity oscillations, which were used for thickness calibration.

The magnetic characterization has been performed in situ using the longitudinal magneto-optic Kerr effect (MOKE) ${ }^{13-15}$ Hysteresis loops were obtained in static magnetic fields up to $B \sim 30 \mathrm{mT}$. With the same optical setup the magnetic susceptibility $\chi(T)$ was studied. For that purpose, the change of the Kerr ellipticity in an applied $a c$ magnetic field has been determined via phase sensitive detection. No additional bias fields were used, hence the zero field $a c$ susceptibility is measured. ${ }^{13}$ In this paper we monitor the static susceptibility, which is obtained for sufficiently low magnetic fields and frequencies. The modulation frequency was set to approximately $110 \mathrm{~Hz}$, while the modulation amplitude $B_{\text {mod }}$ has been varied on purpose. $\chi(T)$ was measured for different in-plane directions of the applied magnetic field, with angular uncertainties of about $\pm 5^{\circ}$. Due to the optical setup we have monitored the magnetic response along the magnetic field direction (longitudinal susceptibility).

Sample heating is a very delicate issue while performing zero-field measurements. At first the heating has to be per- formed quasistatically to achieve an equilibrium phase transition. Secondly, any spurious magnetic fields have to be prevented as they will alter the manifestation of the phase transition, particularly when investigating the zero field susceptibility. For the latter reason we have used an external light source for heating the front side of the sample. Due to this arrangement some scattering of our data appear as the film warms up quicker than the thermocouple, located at the rear side of the sample, on every change of the radiation intensity. This affects the measurements and causes some of the apparently periodic modulations in the plots for $\chi^{-1}(T)$.

\section{THEORETICAL MODEL}

To calculate the susceptibility of the ferromagnetic ultrathin film with an in-plane uniaxial anisotropy we apply an anisotropic Heisenberg Hamiltonian with localized threecomponent spins $\mathbf{S}_{i}$ with spin quantum number $S$ on lattice sites $i$ :

$$
\mathcal{H}=-\frac{1}{2} \sum_{i, j}\left(J_{i j} \mathbf{S}_{i} \cdot \mathbf{S}_{j}+D_{i j} S_{i}^{z} S_{j}^{z}\right)-g \mu_{B} \sum_{i} \mathbf{B} \cdot \mathbf{S}_{i} .
$$

A thin film with $L$ atomic layers is assumed, spanned by the $x z$ plane. $J_{i j}$ is the isotropic exchange interaction between spins $i$ and $j$. The last term is the Zeeman energy, with the magnetic field $\mathbf{B}=\left(B_{x}, 0, B_{z}\right)$ confined to the film plane, where $g$ is the Landé factor and $\mu_{B}$ the Bohr magneton. The uniaxial magnetic anisotropy within a Heisenberg Hamiltonian is usually represented by a magnetocrystalline singleion anisotropy $-\Sigma_{i} K_{2, i}\left(S_{i}^{z}\right)^{2}$, impling a spin quantum number $S \geqslant 1$. However, such a single-ion anisotropy complicates considerably the solution with the method applied in this study ${ }^{16}$ Thus, for simplicity we consider an exchange anisotropy $-(1 / 2) \sum D_{i j} S_{i}^{z} S_{j}^{z}$ between nearest neighbor spins. Although originating from very different physical mechanisms, the anisotropic properties obtained from a single-ion term and an exchange anisotropy are quite similar, if one assumes $K_{2} \sim(q / 2) D$, with $D \equiv D_{i j}$ and $q$ the coordination number. ${ }^{16,17}$ A positive value for $D$ indicates the easy direction to be parallel to the $z$ axis. Note that ferromagnetic thin films with a strong surface anisotropy sometimes exhibit a magnetization perpendicular to the film plane. The interpretation of the susceptibility of such a system is more complicated due to the shape anisotropy resulting from the dipole interaction. The magnetic ground state in this case is a stripedomain structure, and not the single-domain state. ${ }^{18}$ This complication vanishes for an in-plane uniaxial anisotropy, since then the dipole interaction favors a single-domain, ferromagnetic ground state. Thus, this coupling is not considered explicitely in the present study.

The Hamiltonian Eq. (1) is solved by a many-body Green's function approach, ${ }^{3}$ which is suited to consider simultaneously both expectation values $m_{z, i}(T)=\left\langle S_{i}^{z}\right\rangle$ and $m_{x, i}(T)=\left\langle S_{i}^{x}\right\rangle .{ }^{9}$ Furthermore, collective magnetic excitations (spin waves) are taken into account, which are particularly important for low-dimensional systems. The long range magnetic order of an isotropic two-dimensional Heisenberg magnet becomes unstable against collective magnetic excita- 
tions with long wavelengths (Mermin-Wagner theorem). ${ }^{19}$ Already weak anisotropies, however, induce a magnetization with a Curie temperature of the order of the exchange coupling. $^{20}$

We consider the set of anticommutator Green's functions in frequency space $G_{i j}^{\alpha}(\omega)=\left\langle\left\langle S_{i}^{\alpha} ; S_{j}^{-}\right\rangle\right\rangle_{\omega}$, where the ladder operators $S^{ \pm}=S^{x} \pm i S^{y}$ have been introduced and $\alpha=+$, ,$- z$. These Green's functions are solved in the usual way by the equation of motion. ${ }^{3}$ The vanishing eigenvalues occurring in the set of equations motivate the use of anticommutator Green's functions. The higher-order Green's functions appearing within this procedure are approximated by the generalized Tyablikov-decoupling (RPA) for $i \neq k$ (Refs. 3 and 16)

$$
\left\langle\left\langle S_{k}^{\alpha} S_{i}^{\beta} ; S_{j}^{-}\right\rangle\right\rangle \simeq\left\langle S_{k}^{\alpha}\right\rangle\left\langle\left\langle S_{i}^{\beta} ; S_{j}^{-}\right\rangle\right\rangle+\left\langle S_{i}^{\beta}\right\rangle\left\langle\left\langle S_{k}^{\alpha} ; S_{j}^{-}\right\rangle\right\rangle .
$$

Interactions between spin waves are partly taken into account, allowing for the determination of the magnetic properties with a reasonable accuracy in the whole temperature range. It has been shown recently that the magnetization and the Curie temperature of a weakly anisotropic $(S=1)$-Heisenberg monolayer calculated by this approach agrees very well with the values as obtained from a Quantum Monte Carlo method. ${ }^{21}$

The set of linear equations of the corresponding correlation functions $\left\langle S_{j}^{-} S_{i}^{\alpha}\right\rangle$ can be solved numerically for films with an arbitrary number $L$ of layers, for inhomogeneous coupling constants $J_{i j}$ and $D_{i j}$, and for arbitrary spin quantum numbers $S$. This will be investigated in a forthcoming study. ${ }^{9}$ In the remainder of this section we present two cases for which analytical solutions can be derived.

First, to give some insight in the structure of the solutions we consider a homogeneous square (001) ferromagnetic monolayer $\left(L=1\right.$ and $\left.q=q_{\|}=4\right)$ with spins $S=1 / 2$. The coupling constants are put equal to $J_{i j}=J>0$ and $D_{i j}=D$ $>0$ if $i$ and $j$ are nearest neighbors, and zero otherwise. A Fourier transformation into the two-dimensional wave vector space $\mathbf{k} \equiv \mathbf{k}_{\|}=\left(k_{x}, k_{z}\right)$ is applied. By considering the properties for $(S=1 / 2)$-spin operators, the magnetization components $m_{x}(T)$ and $m_{z}(T)$ are given implicitly by the equations

$$
\begin{aligned}
& \frac{1}{2}=m_{z} \frac{1}{N} \sum_{\mathbf{k}} \frac{H}{H_{z}} \operatorname{coth}\left(H / 2 k_{B} T\right), \\
& \frac{1}{2}=m_{x} \frac{1}{N} \sum_{\mathbf{k}} \frac{H}{\widetilde{H}_{x}} \operatorname{coth}\left(H / 2 k_{B} T\right),
\end{aligned}
$$

with the denotations

$$
\begin{gathered}
H=\sqrt{H_{z}^{2}+H_{x} \widetilde{H}_{x}}, \\
H_{z}=m_{z}\left[J\left(q_{\|}-\gamma_{\mathbf{k}}\right)+D q_{\|}\right]+g \mu_{B} B_{z}, \\
\widetilde{H}_{x}=m_{x} J\left(q_{\|}-\gamma_{\mathbf{k}}\right)+g \mu_{B} B_{x}, \\
H_{x}=m_{x}\left[J\left(q_{\|}-\gamma_{\mathbf{k}}\right)-D \gamma_{\mathbf{k}}\right]+g \mu_{B} B_{x},
\end{gathered}
$$

where $\gamma_{\mathbf{k}}=2\left[\cos \left(k_{x} / a_{0}\right)+\cos \left(k_{z} / a_{0}\right)\right], a_{0}$ the lattice constant, and $N$ the number of $\mathbf{k}$ points in the first Brillouin zone. From Eqs. (3) and (4) the susceptibilities $\chi_{z z}$ and $\chi_{x x}$ along the easy and hard axes, which we denote by "parallel" and "transverse" susceptibilities, will be determined numerically. The Curie temperature $T_{C} \equiv T_{C}^{\mathrm{RPA}}$ is calculated from

$$
\frac{1}{4 k_{B} T_{C}}=\frac{1}{N} \sum_{\mathbf{k}}\left[J\left(q_{\|}-\gamma_{\mathbf{k}}\right)+D q_{\|}\right]^{-1} .
$$

Note that the value of $T_{C}$ is determined not only by the isotropic exchange interaction $J$ but depends also on the exchange anisotropy $D .^{16,17,20}$ The mean field approximation (MFA) is obtained by putting $\gamma_{\mathbf{k}}=0$ in Eqs. (6) $-(9)$, yielding the corresponding ordering temperature $4 k_{B} T_{C}^{\mathrm{MFA}}=q_{\|}(J$ $+D$ ). We point out that the paramagnetic Curie temperature $T_{C}^{\text {para }}$ calculated within the Green's function method is equal to $T_{C}^{\mathrm{MFA}} .^{3}$

Secondly, to allow for a quantitative comparison with the $2 \mathrm{ML} \mathrm{Co} / \mathrm{Cu}$ thin film system as investigated experimentally in the present study, we consider a homogeneous fcc (001) film with $L=2$ layers and spins $S=1 / 2$. For a magnetic field along the easy $(z)$ axis $\left[B_{x}=m_{x}(T)=0\right]$ we calculate the magnetization component $m_{z}(T)=m_{z, 1}(T)=m_{z, 2}(T)$ from

$$
\frac{1}{2}=m_{z} \frac{1}{N} \sum_{\mathbf{k}} \frac{\sinh \left(\bar{H}_{\|} / k_{B} T\right)}{\cosh \left(\bar{H}_{\|} / k_{B} T\right)-\cosh \left(m_{z} H_{\perp} / k_{B} T\right)},
$$

denoting

$$
\begin{gathered}
\bar{H}_{\|}=m_{z} H_{\|}+g \mu_{B} B_{z}, \\
H_{\|}=J\left(q_{\|}+q_{\perp}-\gamma_{\mathbf{k}}\right)+D\left(q_{\|}+q_{\perp}\right), \\
H_{\perp}=J \lambda_{\mathbf{k}},
\end{gathered}
$$

where $q_{\perp}=4$ is the coordination number between neighboring layers and $\lambda_{\mathbf{k}}=4 \cos \left(k_{x} / 2 a_{0}\right) \cos \left(k_{z} / 2 a_{0}\right)$. For comparison a simple-cubic (001) film is characterized by $q_{\perp}=\lambda_{\mathbf{k}}=1$. The Curie temperature for this two-layer film is given by

$$
\frac{1}{4 k_{B} T_{C}}=\frac{1}{N} \sum_{\mathbf{k}} \frac{H_{\|}}{\left(H_{\|}\right)^{2}-\left(H_{\perp}\right)^{2}} .
$$

From a fit to experimental data the coupling constants are determined. This can be done by using both $\chi_{z z}(T)$ and $\chi_{x x}(T)$, see Eqs. (3) and (4). Alternatively, one can employ solely $\chi_{z z}(T)$, since the increase of $\chi_{z z}^{-1}(T)$ for $T \gtrsim T_{C}$ depends sensitively on the anisotropy. The latter method is used for the determination of $J$ and $D$ of the present 2 ML case, since for $2 \mathrm{ML}$ an analytical expression is only available for $m_{z}(T)$, see Eq. (10). A corresponding expression for $m_{x}(T)$ along the hard axis needs for additional numerical work.

\section{RESULTS}

The transition temperatures for the $\mathrm{Co} / \mathrm{Cu}(001)$ and the $\mathrm{Co} / \mathrm{Cu}\left(\begin{array}{lll}1 & 1 & 17\end{array}\right)$ thin films exhibit a similar dependence on film thickness. ${ }^{22}$ Due to the high instability against surface 


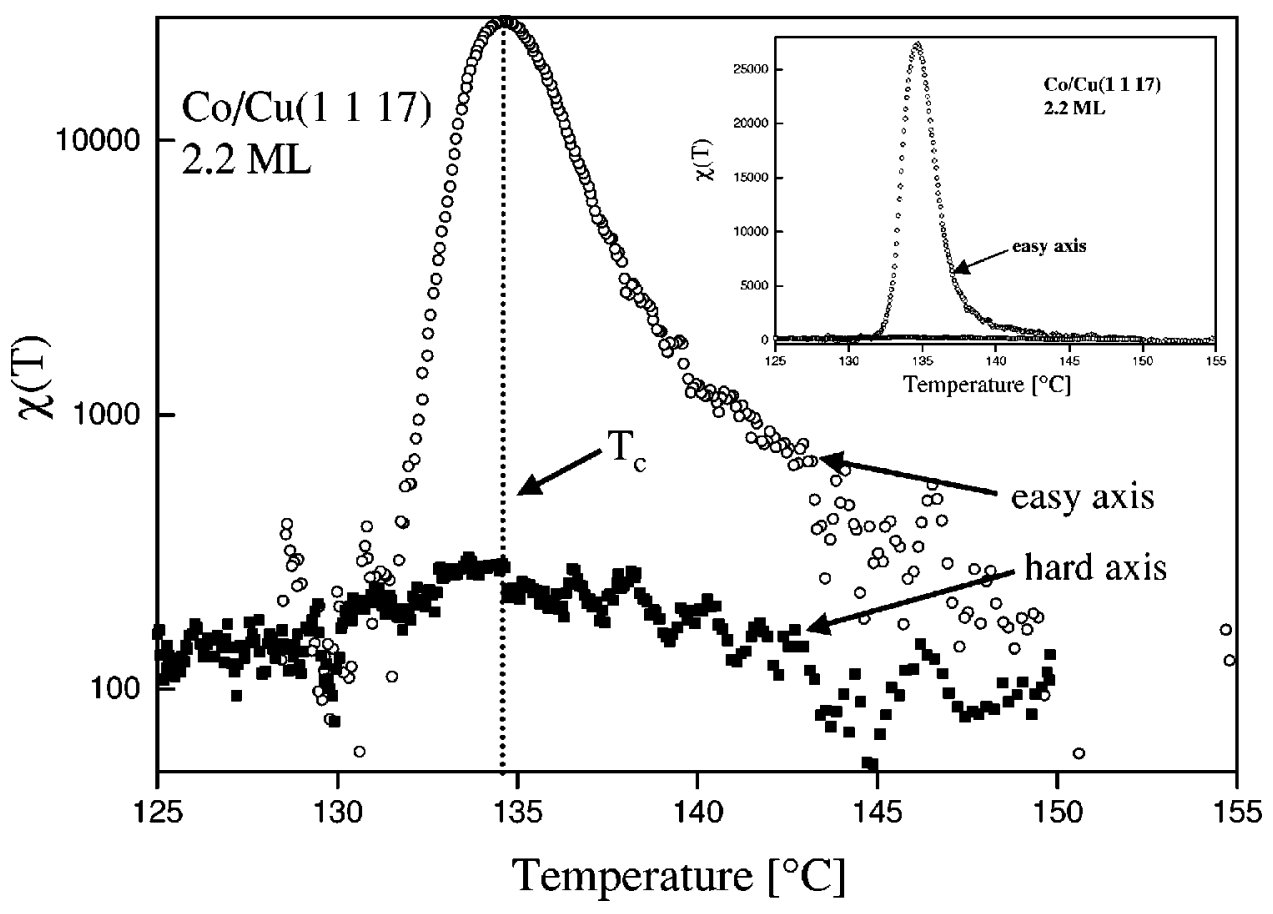

FIG. 1. Parallel and transverse susceptibilities $\chi_{z z}(T)$ (open symbols) and $\chi_{x x}(T)$ (closed symbols) in SI units measured along the easy and hard in-plane directions of a vicinal $\mathrm{Co} / \mathrm{Cu}\left(\begin{array}{lll}1 & 1 & 17\end{array}\right)$ thin film as function of temperature. The nominal film thickness is $2.2 \mathrm{ML}$, the Curie temperature is $T_{C}=134.7^{\circ} \mathrm{C}$. The modulation amplitude is $B_{\bmod }=4.86 \mu \mathrm{T}$ along the easy axis, and $B_{\bmod }=24.3 \mu \mathrm{T}$ along the hard axis, respectively. To present $\chi(T)$ in SI units we have assumed that the saturation magnetization corresponds to the bulk Co value $m_{s}=1.43$ $\times 10^{6} \mathrm{~A} / \mathrm{m}$. A half-logarithmic plot has been used in order to show the temperature behavior of both susceptibilities simultaneously. In the inset the corresponding linear plot is shown.

diffusion of Co films on $\mathrm{Cu},{ }^{23,24}$ and the steep increase of $T_{C}$ with increasing film thickness, ${ }^{25}$ a very thin Co film of about 2.2 ML was chosen with $T_{C}$ well below $180{ }^{\circ} \mathrm{C} .{ }^{23}$ The real part of the susceptibility $\chi(T)$ in SI units as a function of the temperature is shown in Fig. 1. Both parallel and transverse susceptibilities for magnetic field directions along the easy and hard axes are displayed. The semilogarithmic plot allows for a comparison of both quantities. The inset shows the corresponding linear plot. The parallel susceptibility exhibits a strong peak at $T_{C}=134.7^{\circ} \mathrm{C}=407.8 \mathrm{~K}$, with a full width half maximum (FWHM) of $2.7 \mathrm{~K}$ for the actual modulation amplitude $B_{\text {mod }}=4.86 \mu \mathrm{T}$. The FWHM can be reduced to values around $1.5 \mathrm{~K}$ for smaller modulation amplitudes. ${ }^{14}$ For $B_{\text {mod }} \gtrsim 1.62 \mu \mathrm{T}$ an imaginary part of the parallel susceptibility is observed, while for the smallest applied modulation field $\left(B_{\text {mod }}=0.81 \mu \mathrm{T}\right)$ the imaginary part vanishes. ${ }^{22}$

While a peak was found in the susceptibility $\chi_{z z}$ along the easy axis, the magnetic response $\chi_{x x}$ along the hard axis was not detectable for small $B_{\text {mod }} \lesssim 5 \mu \mathrm{T}$. In order to increase the magneto-optical signal and thus the detection limit, the modulation amplitude along the hard axis was increased by a factor of five. However, the response is still small, exhibiting a weak maximum near $T_{C}$, as shown in Fig. 1. Obviously, the phase transition is reflected only weakly in the transverse susceptibility signal. Thus, the phase transition for this thin film system with an in-plane magnetization exhibits a strongly anisotropic behavior.

Figure 2 displays the temperature dependence of the inverse parallel and transverse susceptibilities $\chi_{z z}^{-1}(T)$ and $\chi_{x x}^{-1}(T)$ around the Curie temperature. For elevated temperatures $T \gtrsim 145^{\circ} \mathrm{C}$ the scattering of the data points is strong, and the temperature dependence of $\chi_{z z}^{-1}(T)$ and $\chi_{x x}^{-1}(T)$ cannot be given precisely. As mentioned in Sec. II this scattering is due to the fact that the signal becomes very small, and that the measuring time cannot be increased appropriately as the system properties might change due to the onset of surface diffusion. The oscillations obtained for $\chi_{x x}^{-1}(T)$ are most likely caused by too large steps of changes of the heating power, which were indeed larger than in case of $\chi_{z z}^{-1}(T)$. We have not systematically explored these effects. On the other hand, within the temperature range between $T=T_{C}$ and $T$ $=150{ }^{\circ} \mathrm{C}$ the behavior of $\chi_{z z}^{-1}(T)$ and $\chi_{x x}^{-1}(T)$ is clearly resolved. We remark that the inverse susceptibilities cannot be described by straight lines in this temperature range, rather they exhibit an upward curvature. Evidently, $\chi_{x x}^{-1}(T)$ is shifted upwards with respect to $\chi_{z z}^{-1}(T)$ by an almost constant, temperature independent amount. Thus, at any temperature above $T_{C}$ the inverse susceptibility $\chi_{x x}^{-1}(T)$ along the hard axis is larger than the corresponding value $\chi_{z z}^{-1}(T)$ along the easy axis. This is in accordance with measurements for bulk magnets, ${ }^{6}$ and is also expected theoretically. ${ }^{5} \mathrm{We}$ will show that the temperature range as displayed in Fig. 2 is still far below the linear regime of the inverse susceptibilities.

At first we have carried out corresponding calculations for the parallel susceptibility of a fcc (001) ferromagnetic film with two atomic layers using Eq. (10). The atomic magnetic 


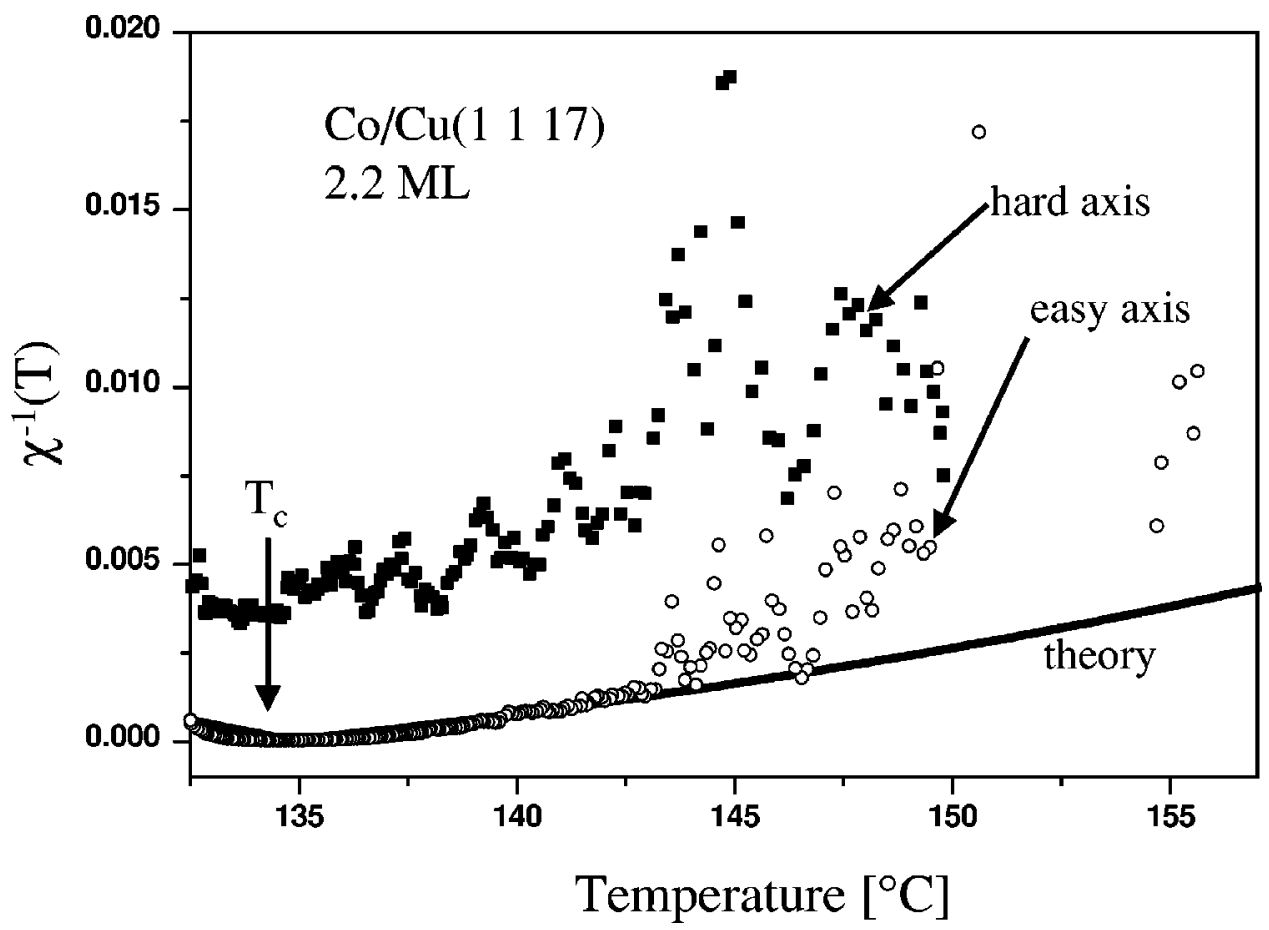

FIG. 2. Inverse susceptibilities $\chi_{z z}^{-1}(T)$ and $\chi_{x x}^{-1}(T)$ in SI-units near the Curie temperature $T_{C}$ $=134.7^{\circ} \mathrm{C}$. The data are taken from Fig. 1. In addition the full line shows $\chi_{z z}^{-1}(T)$ as calculated from Eq. (10), using the isotropic exchange coupling $J=44.1 \mathrm{meV} /$ bond and the exchange anisotropy $D=7.0 \mathrm{mK} /$ atom

moment $\mu_{\mathrm{Co}}=1.7 \mu_{B}$ and the atomic volume $v_{\mathrm{Co}}=1.1$ $\times 10^{-29} \mathrm{~m}^{3}$ appropriate for bulk Co are assumed. The exchange coupling $J$ and the exchange anisotropy $D$ are obtained by fitting the results calculated with Eq.(10) to the measured $\chi_{z z}(T)$ in the temperature range between $T=T_{C}$ and $T=143^{\circ} \mathrm{C}$. We obtain $J S(S+1)=44.1 \mathrm{meV} / \mathrm{bond}$, which is close to the Co bulk value $J_{\mathrm{Co}} S(S+1)$ $=39 \mathrm{meV} /$ bond. Furthermore, we get $D=7.0 \mathrm{mK} /$ atom for the exchange anisotropy, which corresponds to the single-ion in-plane uniaxial anisotropy $K_{2}=(1 / 2)\left(q_{\|}+q_{\perp}\right) D$ $=28 \mathrm{mK} /$ atom $=2.4 \mu \mathrm{eV} /$ atom. From this value the anisotropy energy density is calculated to be $K_{2}^{\prime}=K_{2} / v_{\mathrm{Co}}=3.3$ $\times 10^{4} \mathrm{~J} / \mathrm{m}^{3}$. This value should be compared with the one obtained from independent measurements of the effective anisotropy for the same system. ${ }^{15}$ By means of a thermodynamic perturbation theory ${ }^{4}$ we calculate the microscopic anisotropy energy density at $T=0$ to be $K_{2}^{\prime}=2 \times 10^{4} \mathrm{~J} / \mathrm{m}^{3}$ from the data of Ref. 15, which is in reasonable agreement with the result based on the measured susceptibilities presented here. The resulting small value of $K_{2}^{\prime}$ is comparable to the sixth-order anisotropy energy density $K_{66}^{\text {bulk }}=1.2 \times 10^{4} \mathrm{~J} / \mathrm{m}^{3}$ in the hexagonal plane, and is about 20 times smaller than the second-order uniaxial anisotropy energy density $K_{2}^{\text {bulk }}$ $=76.6 \times 10^{4} \mathrm{~J} / \mathrm{m}^{3}$ of bulk hcp Co. ${ }^{26}$ The calculated inverse parallel susceptibility $\chi_{z z}^{-1}(T)$ is depicted in Fig. 2. A good agreement with experiment is obtained. We note that an increase of the anisotropy $D$ will result in a corresponding decrease of $\chi_{z z}(T)$ for $T>T_{C}$.

We emphasize that the susceptibilities measured in the accessible temperature range up to $155^{\circ} \mathrm{C}$ are still far below the linear (Curie-Weiss) range. As mentioned in the Introduction, the paramagnetic Curie temperature extrapolated from the inverse susceptibilities as calculated by the Green's function method is equal to the Curie temperature $T_{C}^{\mathrm{MFA}}$ obtained from the mean field approximation. ${ }^{3}$ For the parameters $J$ and $D$ as given above we obtain $T_{C}^{\mathrm{MFA}}=1091{ }^{\circ} \mathrm{C}=1364 \mathrm{~K}$. Only for temperatures above $T_{C}^{\mathrm{MFA}}$ a Curie-Weiss behavior will emerge. The difference between $T_{C}$ and $T_{C}^{\mathrm{MFA}}$ and thus the range of the curved behavior of the inverse susceptibilities depicted in Fig. 2 is determined to be very large for this ultrathin film. The reason is that the influence of collective magnetic fluctuations is much stronger in such twodimensional systems as compared to bulk magnets.

Finally, we would like to demonstrate the behavior of $\chi_{z z}^{-1}(T)$ and $\chi_{x x}^{-1}(T)$ in a large temperature range above $T_{C}$. In Fig. 3 the calculated inverse parallel and transverse suceptibilities for a single square ferromagnetic layer are shown, using Eqs. (3) and (4). In order to reveal clearly the difference between $\chi_{z z}^{-1}(T)$ and $\chi_{x x}^{-1}(T)$ a strong exchange anisotropy $D / J=0.05$ is assumed for these calculations. The temperature is given in units of $T_{C}=T_{C}^{\mathrm{RPA}}=0.63 \mathrm{~J}$. For $T>T_{C}$ both susceptibilities differ by a temperature-independent shift, exhibiting the same curvature. The linear behavior of $\chi_{z z}^{-1}(T)$ and $\chi_{x x}^{-1}(T)$ is reached for elevated temperatures $T$ $\gg T_{C}$, where the inverse susceptibilities approach the ones obtained from the MFA. Their extrapolations yield the characteristic temperatures $k_{B} T_{z}=q_{\|}(J+D) / 4$ and $k_{B} T_{x}=q_{\|} J / 4$, which differ by the anisotropy $q_{\|} D / 4$. The larger value $T_{z}$ is the paramagnetic Curie temperature $T_{C}^{\mathrm{para}}=T_{C}^{\mathrm{MFA}}=1.05 \mathrm{~J}$. We point out that the behavior of the inverse susceptibilites as calculated by the MFA is even qualitatively wrong, since it predicts a Curie-Weiss behavior for $\chi^{-1}(T)$ for all temperatures above the Curie temperature.

In contrast, both inverse susceptibilities exhibit a considerably different behavior for $T \leqslant T_{C} \cdot \chi_{z z}^{-1}(T)$ vanishes at $T$ $=T_{C}$ for an infinitely small modulation amplitude, and increases strongly for $T<T_{C}$. On the other hand, $\chi_{x x}^{-1}(T)$ 


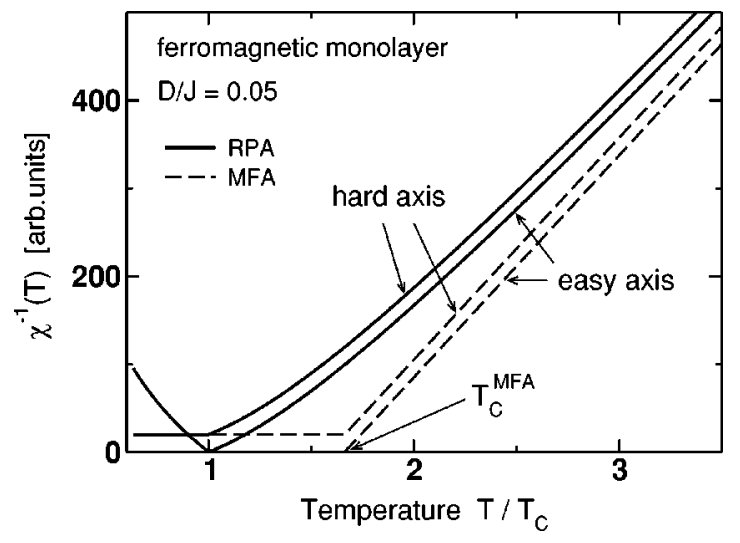

FIG. 3. Calculated inverse parallel and transverse susceptibilities $\chi_{z z}^{-1}(T)$ and $\chi_{x x}^{-1}(T)$ along the easy and hard magnetic directions of a ferromagnetic (001) monolayer as function of temperature (full lines). The calculations have been performed with a Green's function approach (RPA), using Eqs. (3) and (4). For the exchange anisotropy we have assumed $D / J=0.05$, with $J$ the isotropic Heisenberg exchange between neighboring spins. The temperature is given in units of the Curie temperature $k_{B} T_{C} \equiv T_{C}^{\mathrm{RPA}}=0.63 \mathrm{~J}$. For comparison the inverse susceptibilities as obtained from the mean field approximation (MFA) are shown (dashed lines), yielding the Curie temperature $k_{B} T_{C}^{\mathrm{MFA}}=1.05 J$. Here $\chi_{z z}^{-1}(T)$ is depicted only for $T>T_{C}^{\mathrm{MFA}}$.

merely exhibits a cusp at $T_{C}$, and assumes the constant value $\chi_{x x}^{-1}(T)=q_{\|} D$ in the ferromagnetic phase. Thus, one could directly extract the anisotropy $D$ by measuring $\chi_{x x}^{-1}\left(T_{C}\right)$, and determine $J$ from $T_{C}$. This is an alternative treatment to the one as applied for the 2 ML case where both coupling constants are derived from the parallel susceptibility $\chi_{z z}(T)$ alone. However, one has to make sure that secondary processes in the ordered phase are not effective. The apparent peak of the transverse susceptibility observed in Ref. 7 was attributed to vortex and domain wall excitations. These result in a nonconstant behavior of $\chi_{x x}^{-1}(T)$ for $T<T_{C}$, as is also seen in our experiments presented in Fig. 2. For a quantitative comparison such domain processes or multidomain states have to be considered as well. These complications are not expected to occur for $T>T_{C}$.

\section{DISCUSSION AND CONCLUSION}

We have measured the parallel and transverse magnetic susceptibilities $\chi_{z z}(T)$ and $\chi_{x x}(T)$ of a $2.2 \mathrm{ML}$ Co film grown on a vicinal $\mathrm{Cu}\left(\begin{array}{lll}1 & 1 & 17\end{array}\right)$ surface. Corresponding calculations have been performed within an anisotropic Heisenberg model by applying a many-body Green's function method. We have demonstrated that the Curie temperature $T_{C}$ and the susceptibility in the paramagnetic regime ( $T$ $\geqslant T_{C}$ ) gives access to the exchange interaction $J$ and the anisotropy $D$ of ultrathin films.

Using these coupling parameters a quantitative agreement between experiment and theory is obtained at least in the limited temperature range accessible by our measurements. It is evident that this temperature range is far below the cross- over to the Curie-Weiss behavior. Any extrapolation $\chi_{z z}^{-1}(T) \rightarrow 0$ from the experimental data, assuming apparently linear parts in Fig. 2, will yield a wrong value for the paramagnetic Curie temperature $T_{C}^{\mathrm{para}}=T_{C}^{\mathrm{MFA}}$. To reach the linear behavior of the susceptibilities the measurements have to be extended to temperatures around $1100{ }^{\circ} \mathrm{C}$, which is impossible for the $\mathrm{Co} / \mathrm{Cu}$ thin film system due to its instability against surface diffusion and alloying.

The good agreement between theory and experiment justifies a methodological generalization for exploring magnetic properties by investigating the susceptibility in the paramagnetic regime. While the effect of the anisotropy in the paramagnetic regime for bulk systems has been known for a long time, ${ }^{5,6}$ for ultrathin ferromagnetic films improved theoretical approaches considering collective magnetic fluctuations have to be applied. A successful realization of corresponding experiments is challenging as the susceptibilities have to be measured in very small modulation fields with very high accuracy. The measurements should be extended to as large temperatures as possible in order to allow for a comparison with theory over a wide temperature range. From such a comparison values for isotropic exchange interactions and anisotropies as present in the Heisenberg Hamiltonian (1) can be extracted. Note that these quantities refer to constant microscopic parameters and not to effective anisotropies measured at finite temperatures. ${ }^{4}$

In the previous study on $\mathrm{Fe} / \mathrm{W}(110)$ films a quantitative comparison between experiment and theory has not been drawn. ${ }^{7}$ Here a different theoretical approach has been applied, namely, a renormalization group treatment, allowing at the most only for a qualitative comparison. Thus the coupling constants for thin films cannot be determined by this method. It should be mentioned that the Polyakov renormalization scheme applied in Ref. 7 has been strongly criticized. Several authors argue that this scheme might not be applicable for two-dimensional ferromagnets. ${ }^{27}$

In future theoretical work we will explore the behavior of the magnetic susceptibility with increasing film thickness $L$. The range of the curved behavior of $\chi(T)$ for $T>T_{C}(L)$, which is very pronounced for ultrathin films as considered in the present study, is expected to reduce for thick films, approaching the one of the corresponding bulk system. With an improved theory for general spin quantum numbers $S$ the consideration of single-ion anisotropies becomes feasible. Such anisotropies are more appropriate for $3 d$-transition metal magnets. A similar treatment for a perpendicular anisotropy needs the additional consideration of the magnetic dipole coupling. Anisotropies with a different symmetry, e.g., a quartic in-plane anisotropy, are also accessible within the scope of such a treatment, resulting in a considerably different behavior of the susceptibilities with respect to the uniaxial case.

\section{ACKNOWLEDGMENTS}

P.J.J. gratefully acknowledges the invitation and the hospitality of the I. Institute for Theoretical Physics of the Hamburg University. 
*Electronic address: jensen@physik.fu-berlin.de

†Present address: Corporate Research, Thomson Multimedia, DTB, Hermann-Schwer-Str.3, D-78048 Villingen-Schwenningen, Germany.

${ }^{\ddagger}$ Present address: Max-Planck-Institut für Mikrostrukturphysik, Weinberg 2, D-06120 Halle, Germany.

${ }^{\S}$ Electronic address: oepen@ physnet.uni-hamburg.de

${ }^{1}$ See, for example, Ultrathin Magnetic Structures $I+I I$, edited by B. Heinrich and J. A. C. Bland (Springer-Verlag, Berlin, 1994).

${ }^{2}$ W. Wulfhekel, S. Knappmann, B. Gehring, and H. P. Oepen, Phys. Rev. B 50, 16074 (1994); A. Berger, A. W. Pang, and H. Hopster, J. Magn. Magn. Mater. 137, L1 (1994); A. Aspelmeier, M. Tischer, M. Farle, M. Russo, K. Baberschke, and D. Arvantis, ibid. 146, 256 (1995); D. Venus, C. S. Arnold, and M. Dunlavy, Phys. Rev. B 60, 9607 (1999); F. Beusch, G. Garreau, R. Moosbühler, and G. Bayreuther, J. Appl. Phys. 89, 7133 (2001).

${ }^{3} \mathrm{~S}$. V. Tyablikov, Methods in the Quantum Theory of Magnetism (Plenum Press, New York, 1967); W. Nolting, Quantentheorie des Magnetismus (B. G. Teubner, Stuttgart, 1986), Vol. 2.

${ }^{4}$ H. B. Callen and E. R. Callen, J. Phys. Chem. Solids 27, 1271 (1966); Y. Millev and M. Fähnle, Phys. Rev. B 51, 2937 (1995); P. J. Jensen and K. H. Bennemann, in Magnetism and Electronic Correlations in Local-Moment Systems: Rare Earth Elements and Compounds, edited by M. Donath, P. A. Dowben, and W. Nolting (World Scientific, Singapore, 1998), pp. 113-140.

${ }^{5} \mathrm{P}$. Pfeuty and G. Toulouse, Introduction to the Renormalization Group and to Critical Phenomena (Wiley, London, 1977).

${ }^{6}$ H. Suzuki and T. Watanabe, J. Phys. Soc. Jpn. 30, 367 (1970).

${ }^{7}$ C. H. Back, A. Kashuba, and D. Pescia, Phys. Low-Dimens. Semicond. Struct. 2, 9 (1994).

${ }^{8}$ T. Moos, W. Hübner, and K. H. Bennemann, Solid State Commun. 98, 639 (1996).

${ }^{9}$ P. J. Jensen et al. (unpublished).

${ }^{10}$ A. Berger, U. Linke, and H. P. Oepen, Phys. Rev. Lett. 68, 839 (1992).
${ }^{11}$ J. Lapujoulade, J. Perreau, and A. Kara, Surf. Sci. 129, 59 (1983).

${ }^{12}$ J. Frohn, M. Giesen, M. Poensgen, J. F. Wolf, and H. Ibach, Phys. Rev. Lett. 67, 3543 (1991).

${ }^{13}$ A. Berger, S. Knappmann, and H. P. Oepen, J. Appl. Phys. 75, 5598 (1994).

${ }^{14}$ H. P. Oepen, S. Knappmann, and W. Wulfhekel, J. Magn. Magn. Mater. 148, 90 (1995).

${ }^{15}$ W. Wulfhekel, S. Knappmann, and H. P. Oepen, J. Magn. Magn. Mater. 163, 267 (1996).

${ }^{16}$ P. Fröbrich, P. J. Jensen, and P. J. Kuntz, Eur. Phys. J. B 13, 477 (2000).

${ }^{17}$ M. E. Lines, Phys. Rev. B 3, 1749 (1971).

${ }^{18}$ Y. Yafet and E. M. Gyorgy, Phys. Rev. B 38, 9145 (1988).

${ }^{19}$ N. M. Mermin and H. Wagner, Phys. Rev. Lett. 17, 1133 (1966).

${ }^{20}$ C. Herring and C. Kittel, Phys. Rev. 81, 869 (1951); W. Döring, Z. Naturforsch. A 16, 1008 (1961); S. V. Maleev, Zh. Éksp. Teor. Fiz. 70, 2374 (1976) [Sov. Phys. JETP 43, 1240 (1976)]; D. A. Yablonskiy, Phys. Rev. B 44, 4467 (1991).

${ }^{21}$ P. Henelius, P. Fröbrich, P. J. Kuntz, and P. J. Jensen, Phys. Rev. B 66, 094407 (2002).

${ }^{22}$ S. Knappmann, Diplomathesis, RWTH Aachen, Germany, 1994.

${ }^{23}$ A. Schmid, D. Atlan, H. Itoh, B. Heinrich, T. Ichinokawa, and J. Kirschner, Phys. Rev. B 48, 2855 (1993).

${ }^{24}$ M. Giesen, F. Schmitz, and H. Ibach, Surf. Sci. 336, 269 (1995); F. Nouvertné, U. May, M. Bamming, A. Rampe, U. Korte, G. Güntherodt, R. Pentcheva, and M. Scheffler, Phys. Rev. B 60, 14382 (1999).

${ }^{25}$ C. M. Schneider, P. Bressler, P. Schuster, J. Kirschner, J. J. de Miguel, and R. Miranda, Phys. Rev. Lett. 64, 1059 (1990).

${ }^{26}$ J. P. Rebouillat, IEEE Trans. Magn. 8, 630 (1972); D. M. Paige, B. Szpunar, and B. K. Tanner, J. Magn. Magn. Mater. 44, 239 (1984).

${ }^{27}$ A. P. Levanyuk and N. Garcia, J. Phys.: Condens. Matter 4, 10227 (1992); R. P. Erickson and D. L. Mills, Phys. Rev. B 46, 861 (1992). 